\title{
Aplicabilidad del Control Interno en el ejercicio de la Enfermería ${ }^{1}$
}

\author{
Ernestina Aguirre Vidaurre ${ }^{2}$ \\ Yasmín Ramos Cuadra ${ }^{3}$
}

Institución: Clínica Dr. Marcial Fallas, San José.

\section{COMO CITAR}

Aguirre Vidaurre, Ernestina; Ramos Cuadra, Yasmín. Aplicabilidad del control interno en el ejercicio de la Enfermería. Rev. Enfermería Actual en Costa Rica [en línea].201 1, No.20 [citado (fecha)]. Disponible World Wide Web: $<$ http://www.revenf.ucr.ac.cr/aplicabilidad.pdf> ISSN 1409-4568

\section{Resumen}

El objetivo de este artículo es presentar los resultados de una investigación titulada Análisis de los Riesgos en la Gestión de Enfermería Mediante el Control Interno. La población participante correspondió a 9 Profesionales de Enfermería, 23 Auxiliares de Enfermería, 11 Asistentes Técnicos de Atención primaria (ATAPS) y 3 Auxiliares de Enfermería de Centro de Equipo. La investigación fue aprobada por la Escuela de Enfermería de la Universidad de Costa Rica en el año 2006 como trabajo final de graduación. Se desarrolló una metodología cualitativa, aplicando la técnica de la observación para elaborar instrumentos de control interno con el fin de recolectar los datos. Al crear y aplicar dos instrumentos para la solución de problemas se pudo valorar el riesgo en el personal de Enfermería y que repercute en los servicios que brinda. Se concluye que la carencia de personal de enfermería, la infraestructura, el ambiente y clima laboral inadecuado influyen en la prestación de los servicios de salud.

Palabras clave: control-interno, enfermería, gestión, riesgo.

\footnotetext{
${ }^{1}$ Fecha de recepción: 20 setiembre 2010

Fecha de aceptación: 3 de febrero 2011

${ }^{2}$ Profesora de la Escuela de Enfermería de la Universidad de Costa Rica. Máster en Administración Pública. Correo electrónico: ernesagui@hotmail.com

${ }^{3}$ Profesora de la Escuela de Enfermería de la Universidad de Costa Rica. Licenciada en Enfermería. Correo electrónico: yrc08@hotmail.com
} 


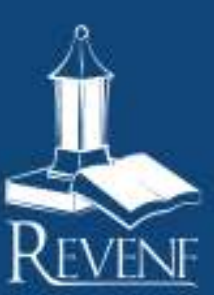

\title{
Applicability of internal control in nursing practice
}

\author{
Ernestina Aguirre Vidaurre \\ Yasmín Ramos Cuadra
}

Institution: Clínica Dr. Marcial Fallas, San José.

\section{CITED}

Aguirre Vidaurre, Ernestina; Ramos Cuadra, Yasmín. Applicability of internal control in nursing practice. Rev.

Enfermería Actual en Costa Rica [en línea].2011, No.20 [citado (fecha)]. Disponible World Wide Web:

$<$ http://www.revenf.ucr.ac.cr/aplicabilidad.pdf> ISSN 1409-4568

\begin{abstract}
The aim of this paper is to present the results of a study entitled Analysis of the Risk Management Internal Control through Nursing. The participant population corresponded to 9 Professional Nursing, 23 nursing assistants, 11 technical assistants Primary Care (ATAPS) and 3 Auxiliary Nursing. This was approved by the School of Nursing at the University of Costa Rica in 2006 as a final work for graduation. We developed a qualitative methodology, using the technique of observation for the development of internal monitoring tools to collect data. Through the creation and implementation of two tools for troubleshooting could assess the risk in the nursing and personal impact on the services provided. We conclude that the lack of nursing staff, infrastructure, environment and inadequate work environment affect the provision of health services as well as recipients.
\end{abstract}

Key words: internal-control, nursing, management, risk.

\section{INTRODUCCIÓN}

En Costa Rica, en el año 2006, la Asamblea Legislativa crea la Ley General de Control Interno: esta ley establece los criterios mínimos que deberán observar la Contraloría General de la República y los entes u órganos sujetos a su fiscalización, en el establecimiento, funcionamiento, mantenimiento, perfeccionamiento y evaluación de sus sistemas de control (Asamblea Legislativa, 2002; p.1). El control interno es un proceso mediante el cual es posible evaluar la labor de una institución respecto de los compromisos que ésta ha adquirido. Dentro de los objetivos que comprende el Control Interno se encuentran la protección del patrimonio nacional, la confiabilidad en los informes de labores, además de la eficiencia y eficacia de las operaciones, así como el cumplimiento de la normativa a la que debe responder la institución. Mediante el control interno, los administradores de las instituciones ejercen la supervisión del trabajo que se realiza, así como su eficiencia por lo que es de suma importancia que se realicen estos controles con la frecuencia y calidad requerida, dado que garantiza la continuidad y eficiencia del sistema de salud de nuestro país. 
Para lograr lo anterior es necesaria la estructuración de programas que tengan como meta principal vigilar la consecución de los objetivos que posee el control interno, por ende, es necesario el desarrollo de instrumentos que permitan el cumplimiento de las normas necesarias de atención.dentro de las instituciones de salud de nuestro país. Al respecto, se sabe que la seguridad en salud en Costa Rica se ha convertido en uno de los baluartes más preciados, lo cual hace de nuestro país uno privilegiado, debido a que muy pocos países a nivel mundial poseen la estructuración operacional y administrativa de nuestro Sistema de Salud actual, la cual se presenta tanto a nivel privado como a nivel público; sin embargo, existen muchos problemas y fallas. (OPS, 2003)

Con el fin de procurar una mejor utilización de los recursos para garantizar su aprovechamiento y uso adecuado y, asimismo, lograr una congruencia entre los objetivos y metas planteados por cada institución es que se ha creado una estrategia de la Gestión de riesgo mediante control interno, lo cual es una forma de garantizar que las instituciones de salud sean responsables del manejo de los recursos con los que cuenta, así como con la obligación que tienen con la comunidad a la cual brinda sus servicios. Tales acciones tienen que ver con los propósitos de la institución, pero la normativa a partir de la cual deben guiarse los funcionarios y funcionarias corresponde a las normativas técnicas, legales y administrativas vigentes.

Es importante tomar en cuenta que el o la profesional de Enfermería como ente gerencial debe dar el paso hacia este tipo de proyectos, pues es una ventana abierta hacia las posibilidades tanto de plazas de trabajo, como de la práctica pura de los conceptos que maneja un profesional de Enfermería desde el inicio de su formación: la Administración de Recursos Humanos en su quehacer diario, la motivación brindada al personal, se convierte en un punto clave del éxito organizacional, aspecto que forma parte del control interno y conforma la estructura de la administración. Esta incluye: "Planificación, organización, formación del equipo de trabajo, dirección y control, elementos esenciales del proceso administrativo de Recursos Humanos " (González, M., 2004; p.87), por lo tanto, al introducir este tipo de proyectos de control de la gestión de Enfermería se puede ver la importancia de que la enfermera o enfermero sea quien lo lleve a cabo, ya que por su formación y responsabilidades dentro de un Centro de Salud, es el profesional idóneo para lograr la consecución de objetivos.

Aguirre (2010) menciona que la gestión es una herramienta en enfermería que ha permitido la formación de equipos de alto desempeño, un entorno seguro, la atención de salud.la delegación y la supervisión. (Aguirre, 2010, p.81). Por lo tanto, es importante que la persona gestora guíe su actividad a partir de sus conocimientos para obtener resultados positivos en todo lo que se refiere al control interno.

El objetivo de este estudio fue analizar el riesgo en la gestión de Enfermería mediante el Control Interno en la Clínica Dr. Marcial Fallas Díaz, durante los meses de marzo a julio del año 2005.

\section{MATERIALES Y MÉTODOS}

La población participante estuvo conformada por 9 profesionales de Enfermería (1 directora, 3 del área de emergencias, 3 en el primer nivel, 1 obstetra y 2 de salud mental; 23 Auxiliares de Enfermería, 11 Asistentes Técnicos de Atención primaria (ATAPS) y 3 Auxiliares de Enfermería del Centro de Equipo, cuya selección fue por conveniencia (Barrantes, 2000), debido a la rotación y turnos vespertinos.

Por otra parte, es un estudio descriptivo en el que se empleó la técnica de observación, así como el elaborar 


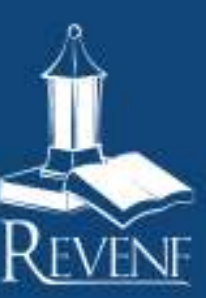

\section{Revista Electrónica Enfermeria Actual en costa Rica}

instrumentos de control interno utilizados como los principales métodos de recolección de datos.

Los instrumentos elaborados para este trabajo fueron:

Instrumento para la identificación y evaluación del riesgo en las actividades que realiza el personal de enfermería. Este instrumento fue aplicado a los participantes seleccionados. Cada instrumento se dividió en cinco apartados según las funciones del profesional de Enfermería, las cuales son: en su grado personal y profesional en Planificación, Organización, Dirección y Control; congruentes con las etapas del control interno.

Para el resto del personal se tomaron en cuenta las actividades que deben realizar de acuerdo con su puesto. La máxima puntuación alcanzada será considerada excelente y dependiendo del puntaje que obtenga podrá ser calificado como muy bueno, bueno y regular.

Instrumento para la identificación del ambiente y clima laboral del personal de enfermería. Este instrumento fue construido con el fin de determinar cuáles condiciones de trabajo conllevan algún grado de riesgo que sitúe en peligro no sólo la atención a los usuarios, sino también al personal de enfermería. Parte de identificar y evaluar el riesgo en el ambiente y el clima laboral, factores importantes para el desempeño de los profesionales de Enfermería en un centro de salud.

El instrumento tuvo una escala con las siguientes calificaciones: MB (Muy buena) 3 puntos, B (Buena) 2 puntos, R (Regular) 1 punto y M (Mala) 0 puntos. Muy buena es la mayor calificación y, mala, la más baja. Además, constó de dos partes: una primera parte fue el Ambiente Labora, la cual consta de 5 ítems cuya calificación más alta fue de 15 puntos y la más baja de 0 puntos. En este punto se evalúa el lugar de trabajo, a partir de la opinión del personal de enfermería, en donde se desarrollan las acciones de Enfermería o pasan la mayor parte del tiempo laborando. Esta categoría incluye ventilación, iluminación, espacio de trabajo, orden e higiene. La segunda parte evaluó el Clima Laboral: con 7 ítems cuya calificación más alta fue 21 puntos y la más baja 0 puntos. Se tomó en cuenta el factor humano, es decir, la manera en que el personal de Enfermería percibe a sus compañeros y el campo de trabajo; dentro de los elementos contemplados están la motivación personal, las asignaciones de responsabilidades, los espacios de recreación, el estrés, los programas educacionales, cursos de refrescamiento y horarios tanto de comida como de descanso.

Finalmente, se realizó el análisis de los resultados, priorizando aquellas condiciones que fueron de mayor importancia para un mejor desempeño del personal de Enfermería.

\section{Consideraciones éticas.}

Respecto de los fundamentos éticos, se contó con un documento que contenía la descripción del estudio, objetivo, beneficios y garantía de total confidencialidad; éste fue presentado ante la dirección de enfermería del centro de salud donde se llevó a cabo la investigación dándose y así se obtuvo el aval para el desarrollo de los objetivos. Además, las investigadoras se aseguraron de no utilizar nombres, iniciales o números que pudiesen identificar a las personas participantes. 


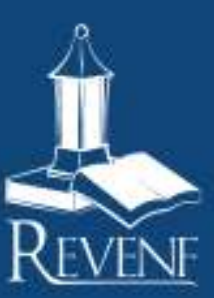

\section{Revista Electrónica Enfermeria Actual en Costa Rica}

\section{RESULTADOS}

Asistente Técnico de Atención Primaria (ATAP): ninguno de los ATAP llegó a la categoría de excelencia en el desempeño de sus funciones; sin embargo, 4 personas se encuentran en los rubros de muy bueno y bueno, lo que ejemplifica que el trabajo que se lleva a cabo puede cumplir con las metas del Plan Operativo Anual. Dos alcanzaron una calificación de regular y, uno, se mantuvo en el rubro de malo.

Respecto de la infraestructura, el local en donde funciona el Centro de Salud cuenta con un espacio dispuesto para oficina y bodega, con un área muy reducida para un adecuado funcionamiento. Además, la ventilación e iluminación no son eficientes para la cantidad de personas que permanecen allí, lo que constituye un riesgo en la salud laboral.

En cuanto a la atención directa, los ATAP refieren que:

"no se puede brindar una atención de calidad, pues dicen que en una misma familia se encuentran polipatologías que deben ser captadas en forma individual, por lo que se requiere de mayor tiempo para realizarlas y no del que disponen generalmente, además, las referencias realizadas a los diferentes servicios de la Clínica, no son tomadas en cuenta, lo cual afecta la credibilidad de su trabajo dentro de la comunidad y la participación que se pueda tener".

Los ATAP refieren sentirse poco motivados pues aseguran que "los estímulos son pocos y no hay relación directa con la jefatura. Además, por las condiciones laborales en las que se encuentran", otro aspecto que señalaron fue que "no se valora el trabajo que realizamos, y que además no hay trabajo en equipo en las jefaturas". Otro de los puntos que consideran les afecta es la escasa educación continua, la cual hasta hace poco se está brindando; sin embargo, les gustaría que se tomara en cuenta temas de su competencia directa y les ayuden en su atención a la comunidad. Al respecto, una de las ATAP refiere que "lo que se vaya a dar de educación debe ser relacionado con nuestro trabajo y de nuestro interés",

Auxiliares de Enfermería: 16 de ellos y ellas tienen muy buen desempeño y 7 evidenciaron un buen desempeño en todas las acciones que ejecutan.

La planta física en donde labora el personal auxiliar, en el área de emergencias, no es adecuada para la atención de las diferentes afecciones que aquejan a los usuarios de este servicio, además, los cubículos individuales son muy pequeños e incómodos. No hay una visualización de las personas ingresadas desde el puesto de Enfermería. Por otro lado, la ventilación es inadecuada, el espacio es reducido, un ejemplo es que el introducir una camilla a la sala de "shock" resulta sumamente incómodo. La rampa no cumple con las medidas reglamentarias; no se cuenta con salidas de emergencia y no existen las condiciones mínimas para manejar la técnica aséptica médica, ya que no hay jaboneras, ni dispensador de servilletas.

Otro sitio observado fue la planta física de medicina 1 y 2, la cual no cuenta con suficiente espacio, una adecuada ventilación ni privacidad necesaria para llevar a cabo las actividades diarias de las preconsultas de Enfermería. El lavado de manos no se realiza después de la atención a las personas, debido a la carencia de lavatorio, jabón y dispensadores. 


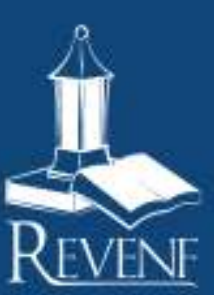

\section{Revista Electrónica Enfermeria Actual en costa Rica}

La pileta que existe para hacer las curaciones es poco adecuada, por tanto, impide el desempeño correcto del personal que opta por no utilizarla la mayoría de las veces. No se cuenta con suficiente material estéril para realizar los procedimientos de curaciones. Otro factor importante que se observó es que el personal no realiza notas de enfermería en la preconsulta.

En cuanto a la educación continua, fue evidente que no se desarrolla el programa en forma efectiva de acuerdo con las necesidades y prioridades del personal. Además, no se tiene tiempo asignado para asistir a las capacitaciones. Se encontró que el estrés laboral es un factor que incide negativamente en el buen funcionamiento de las tareas del personal auxiliar.

Personal de Centro de Equipos: este personal demostró un excelente desempeño en las labores que realiza.

Enfermera Obstetra: como profesional especialista es un recurso que esta subvalorado. Las funciones de la enfermera obstetra son muy limitadas y su experiencia y conocimientos no están siendo aprovechados al máximo.

Enfermeras que laboran en el área de emergencias: el desempeño laboral de dos de ellas se encuentra en el rubro de bueno y una en el rubro de regular. La sobrecarga de funciones dificulta la atención oportuna, personalizada y de calidad que se pueda brindar a las personas, a pesar de los esfuerzos que realiza el personal.

Enfermera que labora en el primero y segundo nivel: dos de ellas obtuvieron en el desempeño un puntaje de excelente y, una, de bueno, este aspecto puede afectar una buena calidad de atención en el primer nivel. Las enfermeras señalaron que tienen sobrecarga laboral y que muchas veces hay dualidad en las funciones.

Enfermera de Salud Mental: el desempeño de una de las enfermeras se evaluó como excelente y otra de ellas obtuvieron un puntaje que las coloca en el rubro de malo.

Dirección de Enfermería: la enfermera que labora en la dirección evidenció que la mayoría de las actividades una evaluación entre excelente, muy bueno y bueno. En cuanto a las funciones de educación, participación social y conjunta, muchas veces es difícil de cumplir, pues existen funciones administrativas y operativas que tienen una mayor prioridad.

En la tabla 1 se evidencia los hallazgos más relevantes.

\section{Tabla 1}

Clínica Dr. Marcial Fallas: Problemas encontrados en el personal de Enfermería según el Control Interno, 2010.

\begin{tabular}{l|l}
\hline \multicolumn{1}{c|}{ Problemas Encontrados } & \multicolumn{1}{c}{ Personal de Enfermería } \\
\hline Déficit en la calidad de atención al usuario. & $\begin{array}{l}\text { AT APS,-Auxiliares de } \\
\text { enfermería-Enfermeras }\end{array}$ \\
\hline No hay participación comunitaria & $\begin{array}{l}\text { AT APS-Auxiliares de } \\
\text { enfermería -Enfermeras }\end{array}$ \\
\hline
\end{tabular}




\begin{tabular}{l|l}
\hline Sobrecarga de trabajo & $\begin{array}{l}\text { Auxiliares de enfermería - } \\
\text { Enfermeras- Directora de } \\
\text { Enfermería }\end{array}$ \\
\hline $\begin{array}{l}\text { Deficiencias en la utilización de la técnica } \\
\text { aséptica medica y quirúrgica }\end{array}$ & $\begin{array}{l}\text { ATAPS-Auxiliares de } \\
\text { Enfermería, Enfermeras }\end{array}$ \\
\hline Manejo de Equipos deficiente & $\begin{array}{l}\text { Auxiliares de enfermería- } \\
\text { Enfermeras }\end{array}$ \\
\hline No hay Supervisión continua & $\begin{array}{l}\text { Directora de Enfermería- } \\
\text { Enfermeras }\end{array}$ \\
\hline No hay una educación permanente & $\begin{array}{l}\text { ATAPS- auxiliares de } \\
\text { enfermería-misceláneos de } \\
\text { centro de equipo }\end{array}$ \\
\hline
\end{tabular}

\section{DISCUSION}

Esta investigación representa la capacidad administrativa del profesional de Enfermería y de la necesidad de implementar auditorías de Enfermería dentro del sistema de salud costarricense, las cuales produzcan un mejor manejo de los recursos humanos, una atención de mayor calidad y más calidez en los centros de salud. El Control Interno puede brindar las herramientas necesarias para lograr un manejo adecuado de los sistemas administrativos de los servicios de salud y, así, solucionar las oportunidades de mejora que puedan encontrarse.

Es evidente que mediante el control interno puede ser visible la motivación del personal, factor determinante para el buen funcionamiento humano. La importancia recae en que todas las personas que trabajan dentro de una organización se encuentran tras varios objetivos, los cuales deben ser llenados por el trabajo que realicen, de lo contrario afectará eventualmente la producción o el servicio que se brinde. Se debe tomar en cuenta varios factores, no solamente señalarle a la persona que su trabajo es eficiente y otorgarle una remuneración. $\underline{\text { Chiavenato }}$ (1999) menciona que "existen varias condiciones necesarias para que exista un buen desempeño: higiene laboral, salud ocupacional, disminución del estrés laboral, estabilidad laboral, salud y beneficios, deporte y recreación y programas participativos" ( p.475)

Todos estos aspectos deben introducirse en la administración diaria del personal, así, se contará con personas trabajadoras motivadas que ejecuten sus funciones para alcanzar los objetivos de la empresa, ya que sus necesidades físicas y mentales han sido concretadas.

Tampoco se debe olvidar que en la profesión de Enfermería siempre se trabajará con personas que tienen sentimientos, diferentes caracteres y pensamientos, cambios de humor, se sentirán tristes o alegres, estarán enfermos o se sentirán bien; lo importante es siempre tener buenas relaciones interpersonales, e incorporar en nuestra forma de vida la asertividad y tratar de ser gerentes negociadores. La motivación del personal a cargo resulta ser una necesidad primordial en el trabajo de un buen gerente, pues "la motivación hace que el rendimiento en el trabajo sea óptimo, que las personas se sientan mejor consigo mismas y con los demás y les hace sentir necesarias y útiles en su trabajo" (Potter y Perry, 2000; p.67), todo lo anterior incrementa la productividad y la calidad de los servicios que se brinda. 
También la seguridad de las personas tiene un papel importante en el ambiente laboral, ya que se relaciona con la atención libre de riesgo que se exige para las clínicas dentro del compromiso de gestión y control interno (Ladino, E., 2001), por lo cual se deben habilitar las salidas de emergencia y los espacios de salida, el aseo de los pasillos, la señalización y el conocimiento de los planes de emergencia en los servicios, puesto que tales mejoras evitarán futuros problemas en caso de que haya algún accidente o catástrofe.

Todo lo mencionado es fundamental para lograr que una empresa se desarrolle de manera efectiva; sin embargo, se debe tomar en cuenta el trabajo que realizan todos, debido a que "todos los trabajadores constituyen un engranaje que mueve la máquina empresarial” (Dressler. G., 1999; p. 102), lo cual resalta la importancia de tomar en cuenta que todas las personas que trabajen en una organización deben tener la adecuada capacitación según su desempeño.

El control interno demanda que existan supervisiones cuyo alcance y frecuencia esté determinado por la naturaleza e importancia de los riesgos que generen las intervenciones que se realizan (Mejía, B., 1999). Por lo tanto, cuando los profesionales no asumen sus funciones, hay un gigantesco desperdicio de recursos, pues por ley debería tener tanto el derecho como el deber de ejercer su profesión de manera completa (Colegio de Enfermeras de CR. 2005).

Actualmente, la proyección a la comunidad es una necesidad en salud, así lo dice Gómez en Control Interno, quien considera que el control interno es una responsabilidad de todos los integrantes de la organización empresarial, es decir, "la nueva línea de prevención de la enfermedad y la promoción de la salud debe ser fuera de los centros de salud" (Gómez, G., 2004; p. 56), con el fin de que los profesionales se integren de manera efectiva en el medio en que viven las comunidades y así las intervenciones serán más próximas a la realidad de las personas.

\section{CONCLUSIONES}

Se requiere de mayores recursos humanos, principalmente de:

- Un profesional de Enfermería coordinador (a) del equipo básico de atención integral de salud (ATAPS).

- Contratación de ATAP para lograr alcanzar los objetivos de cobertura.

- Un profesional de Enfermería en educación en servicio que capacite constantemente al personal y un supernumerario para los servicios en saturación.

- La sobrecarga laboral en la dirección de enfermería amerita asignar una subdirección de Enfermería para que el trabajo pueda delegarse y hacer más eficiente el desempeño del proceso administrativo.

El escaso trabajo en equipo dificulta las funciones del personal de Enfermería. Por otra perte, la comunicación interna, tanto del personal técnico como profesional, es relevante para que las diferentes funciones sean de calidad.

La iluminación, la ventilación y el espacio son factores de suma importancia que las instituciones deben tener para que el personal y los usuarios estén en un ambiente libre de riesgo, por lo tanto, es responsabilidad de la 


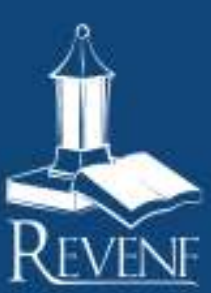

\section{Revista Electrónica Enfermeria Actual en Costa Rica}

administración tener una infraestructura adecuada para atender al público y para que las personas que trabajan en estos servicios puedan desempeñarse correctamente.

Las funciones de las enfermeras de salud mental abarcan, entre otras, participar en programas participativos dirigidos a todo el personal de enfermería y velar por la salud ocupacional en el ámbito mental.

El control interno demanda la existencia de supervisiones cuyo alcance y frecuencia esté determinado por la naturaleza e importancia de los riesgos que generen las intervenciones que se realizan.

\section{BIBLIOGRAFÍA}

Aguirre, E. (2010).Pautas para el fortalecimiento continuo en la gestión de la Enfermería. San José: Uruk Editores.

Asamblea Legislativa de la República de Costa Rica. (2002). Ley General de Control Interno. San José, Costa Rica.

Barrantes, R. (2000). Investigación: Un Camino al Conocimiento, un Enfoque Cualitativo y Cuantitativo. San José: UNED

Chiavenato, I. (1999) Gestión del Talento Humano. México D.F.:Mc Graw Hill.

Colegio de Enfermeras de CR. (2005) Reglamento de enfermería Ginecostétrica y perinatal de Costa Rica. San José, C.R.: La Gaceta 10

Dressler. G. ( 1999) Administración de Personal. N.Y: Prentice Hall. Hispanoamericana S.A

Gómez, G. (2004). Control Interno: Una responsabilidad de todos los Integrantes de la organización empresarial. México D. F.: Editorial Gestiópolis.

González, M. (2004) Manual de Administración de Recursos Humanos. San José: UACA

Ladino, E. (2001). Normas Generales de Control Interno, Normas de Ambiente de Control e Integridad y Valores Éticos. San José: UNED

Mejía, B. (1999). Gerencia de Procesos para la organización y el Control interno de empresas de salud. Bogotá: Ecoe ediciones Organización Panamericana de la Salud, 2003. Desigualdades de Salud en Costa Rica. San José, Costa Rica Ministerio de Salud. Potter y Perry, P. (2000.) Fundamentos de Enfermería Vol I. IV Edición. Madrid. Editorial Océano. 


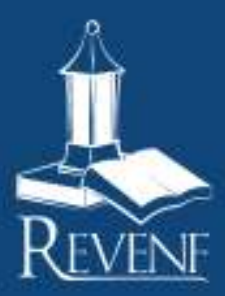

